\title{
Verification of Signal-to-Crosstalk Measurements for WDM Fiber Optical Parametric Amplifiers
}

\author{
Áron D. Szabó ${ }^{*}$, Vitor Ribeiro ${ }^{1}$, Vladimir Gordienko ${ }^{1}$, Filipe Ferreira ${ }^{1,2}$, Chandra Gaur $^{1}$ and $^{\text {Nick Doran }}{ }^{1}$ \\ ${ }^{\prime}$ Aston Institute of Photonic Technologies, Aston University, Birmingham, B4 7ET, UK \\ ${ }_{2}^{2}$ now with University College London, Gower Street, London, WC1E 6BT, UK \\ *a.szabo1@aston.ac.uk
}

\begin{abstract}
Gaussian distribution of nonlinear inter-channel crosstalk noise is numerically shown in fiber optical parametric amplifiers with over $16 \mathrm{~dB}$ gain. Confidence of signal-to-crosstalk power ratio measurements is justified by consistency with error vector magnitude calculations. (C) 2020 The Authors
\end{abstract}

\section{Introduction}

Nonlinear inter-channel crosstalk due to four-wave mixing poses performance limitations on WDM transmission systems as well as on fiber optical parametric amplifiers (FOPAs). Compensation techniques in the digital and optical domain are investigated extensively for WDM transmission systems [1], while optical compensation techniques are more pronounced for FOPAs, for example proper positioning of the gain and idler removal stages in a polarizationinsensitive FOPA [2] or realizing a Raman-assisted FOPA [3]. Detrimental crosstalk in any WDM FOPA needs to be overcome in order to exploit their practical potential, including gain bandwidth over $100 \mathrm{~nm}$ or ultra-low noise multichannel amplification as a phase sensitive amplifier [4].

Towards this end it is crucial to understand the characteristics and measurement of accumulated noise due to nonlinear processes including inter-channel interference. It has already been shown by Carena et al. [5] that polarization-multiplexed QPSK signal components become Gaussian-distributed following 100-5000 km propagation in dispersion uncompensated optical links even in the absence of added amplified spontaneous emission (ASE) along the link. However, to the best knowledge of the authors, no similar investigation has been performed for FOPAs, which regularly operate in the strongly nonlinear regime and with close to negligible dispersion in the gain band.

In this summary, we show for the first time to our best knowledge that inter- and intra-channel nonlinear effects in a WDM FOPA generate Gaussian-distributed noise in the channels even in the absence of ASE. The result is consequently used to show the consistency between measurable signal-to-crosstalk power ratio and error vector magnitude $(\mathrm{EVM})$. The revealed connection justifies the confidence of practical signal-to-crosstalk power measurements in quantifying signal degradation due to nonlinear crosstalk.

\section{Calculation methods}

Signal-to-crosstalk power ratio is regularly determined in practice by leaving the investigated WDM channel empty and measuring the generated crosstalk product (Fig. 1). FOPAs operate in the highly nonlinear regime where the investigated channel itself highly contributes to the process and signal and idler phases are correlated. In order to justify the confidence of the method for this regime, we show the relationship between signal-to-crosstalk power ratio and EVM without the inclusion of ASE, characterizing the effects of solely nonlinearities and dispersion on the signal quality. For $x$ QAM constellations where $\log _{2}(x)$ is even including QPSK, the EVM and OSNR are directly related and the $O S N R \approx E V M^{-2}$ approximation is used [6], supposing the nonlinear noise is Gaussian distributed.
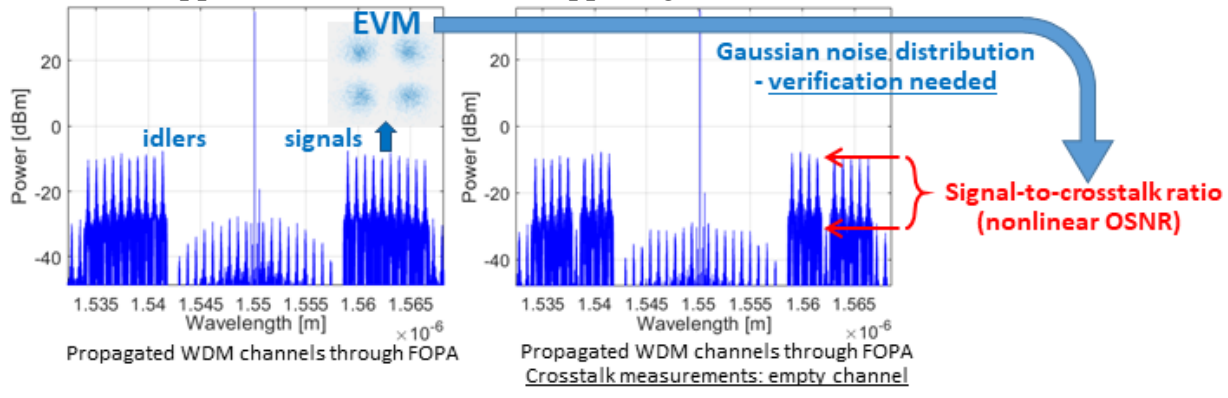

Figure 1: Verification of signal-to-crosstalk power ratio measurements for FOPA

In order to prove Gaussian distribution of the nonlinear noise for scenarios of different channel spacing and input signal power, the coupled nonlinear Schrödinger equations [7] considering four-wave mixing, self-phase modulation, cross-phase modulation and dispersion are solved using the split-step Fourier method for the pump and the modulated signal waves along the FOPA. Propagation along a single polarization state of the highly nonlinear fiber (HNLF)- 
based FOPA is assumed. With the pump placed at the zero dispersion wavelength, dispersion terms of $\beta_{2}=0, \beta_{3}=$ $0.07 \frac{\mathrm{ps}^{3}}{\mathrm{~km}}$ and $\beta_{4}=10^{-4} \frac{\mathrm{ps}^{4}}{\mathrm{~km}}$ are used. The FOPA consisted of $200 \mathrm{~m}$ of HNLF with $\gamma=0.014(\mathrm{Wm})^{-1}$ and $35 \mathrm{dBm}$ pump was applied. $50 \mathrm{Gbps}$ per polarization QPSK signals are transmitted for each scenario of parameters, equivalent to a realistic 27.75 GBaud, $11 \%$ overhead for FEC and line coding [5]. Calculations of output signal powers for each scenario were based on up to 50 realizations of $\left(2^{8}-1\right)$-long independently randomized bit sequences together with independently randomized initial phases to consider the finite coherence time of the transmitters. Experimental validation of the split-step solver was demonstrated in a preliminary work for crosstalk calculation in FOPA [8].

\section{Results and discussion}

Calculations were performed for multiple FOPA scenarios of 10 WDM channels with spacing of 50, 100, $150 \mathrm{GHz}$ and $-10 \mathrm{dBm}$ per channel input, as well as with $100 \mathrm{GHz}$ channel spacing and $-20,-15,-10,-5 \mathrm{dBm}$ per channel input, 2 scenarios of which are shown in Fig. 2. Proper guard band was applied to avoid crosstalk products around the pump as shown in Fig. 1. Gaussian distribution of the generated nonlinear crosstalk noise without added ASE is confirmed for both constellation components over the investigated parameter range, examples of which are shown in Fig. 2. High confidence of $R^{2}>98.2 \%$ was found even for the highest signal power and more typically $R^{2}>99 \%$.
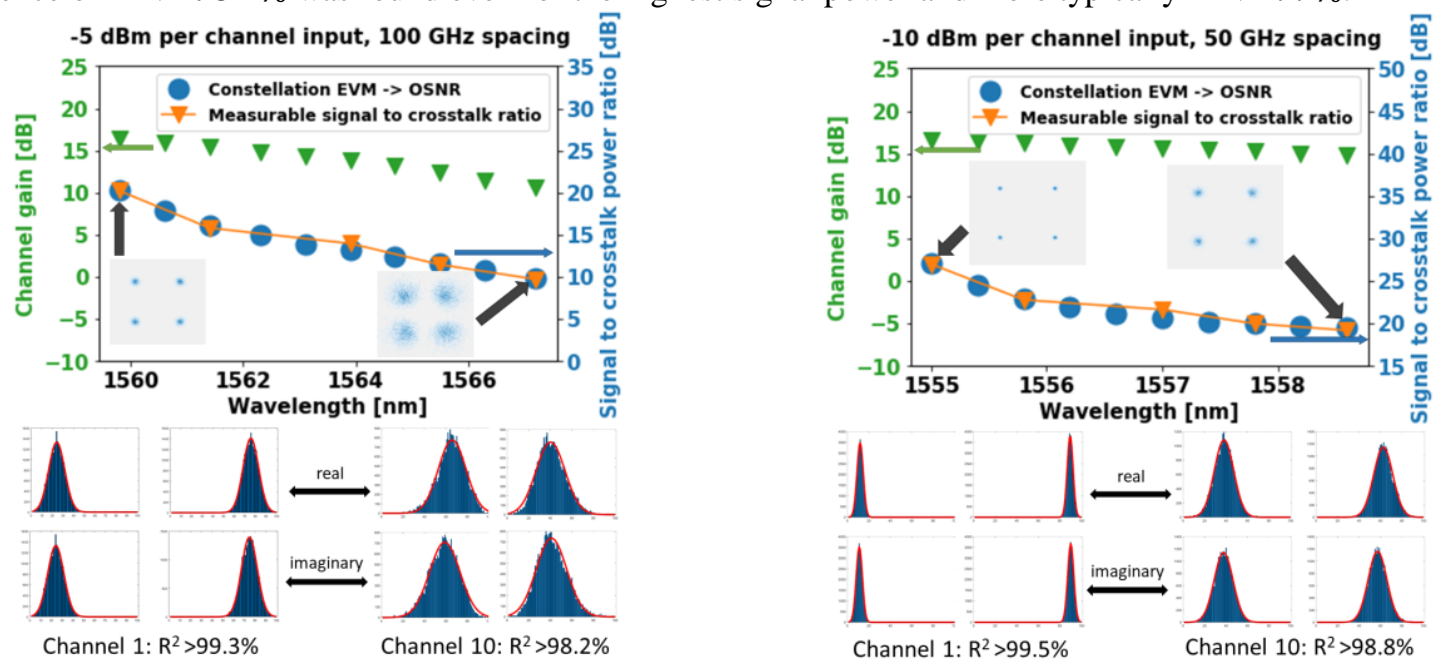

Figure 2: Consistency of measurable (empty channel) and EVM-related signal-to-crosstalk power ratios; gain and Gaussian nonlinear noise fitting

Signal-to-crosstalk power ratio decreases towards longer wavelengths as channel gain decreases due to the dispersion of the HNLF. In the above parameter range as well as for repeated calculations with $30 \mathrm{dBm}$ pump power, the signal-to-crosstalk power ratio calculated from EVM shows excellent match with the measurable signal-tocrosstalk power ratio calculated using the empty channel method, regardless of the gain variation as shown in Fig. 2.

\section{Conclusion}

In this paper we have shown that nonlinear crosstalk and distortion in WDM FOPAs produce Gaussian distributed noise even in the absence of ASE for input signal powers between $-20 \mathrm{dBm}$ and $-5 \mathrm{dBm}$ with $35 \mathrm{dBm}$ pump power and with $50-150 \mathrm{GHz}$ channel spacing. Signal-to-crosstalk power ratio is verified as an accurate quantity to describe nonlinear degradation of WDM QPSK signal channels by showing excellent agreement with EVM calculations.

\section{Acknowledgement}

The authors would like to thank Dániel Mazroa (formerly with Budapest University of Technology and Economics) for valuable discussions on the applied numerical model. The work was funded by EC Marie-Curie MULTIPLY projects OPERNET and POLSAR, as well as by EPSRC projects UPON (EP/M005283/1), FOPA-ROCS (EP/R024057/1) and PEACE (EP/L000091/1). The data reported in this paper is available at https://doi.org/10.17036/researchdata.aston.ac.uk.00000450 as part of the UK EPSRC open access policy.

\section{References}

[1] V. Vgenopoulou, M. Song, E. Pincemin, Y. Jaouën, S. Sygletos and I. Roudas, Appl. Sci. 8, 447 (2018)

[2] V. Gordienko, F. Ferreira, A. Szabo, V. Ribeiro, C. Laperle, M. O'Sullivan, K. Roberts, A. Ellis and N. Doran, ECOC paper Th.1.C.5. (2019)

[3] A. Redyuk, M.F.C. Stephens and N. J. Doran, Opt. Exp. 23, 27240-27249 (2015)

[4] M. E. Marhic, P. A. Andrekson, P. Petropoulos, S. Radic, C. Peucheret and M. Jazayerifar, Las. \& Photon. Rev. 9, 50-74 (2015)

[5] A. Carena, G. Bosco, V. Curri, P. Poggiolini, M. Tapia Taiba, F. Forghieri, ECOC paper P4.07 (2010)

[6] W. Freude et al., ICTON paper Mo.B1.5 (2012)

[7] G. Agrawal, Nonlinear Fiber Optics (5 ${ }^{\text {th }}$ edition, Academic Press, 2013), Chap. 10.

[8] Á. Szabó, B. J. Puttnam, D. Mazroa, A. Albuquerque, S. Shinada, and N. Wada, Photon Technol. Lett. 26, 1503-1506 (2014) 